\title{
Combining 308-monochromatic excimer phototherapy with monthly IM triamcinolone acetonide for the treatment of resistant alopecia totalis
}

\author{
Nabeel K. Alhamzawi
}

Department of Dermatology, Diwaniyah Teaching Hospital, Diwaniyah, Iraq

\begin{abstract}
Background: Treatment of resistant alopecia totalis AT is a major problem in general practice. Some studies reported the use of either excimer-308 or intra-muscular triamcinolone acetonide as a monotherapy, with conflicting results.

Objective: To evaluate the therapeutic effect of combining 308-excimer phototherapy and intramuscular triamcinolone acetonide for the treatment of alopecia totalis.
\end{abstract}

Methods and Material: Ten patients with alopecia totalis were evaluated in this prospective interventional study. All patients were assigned to receive the thera-peutic regimen that includes monthly IM triamcinolone acetonide (TAC) for a maximum of six pulses and twice-weekly excimer phototherapy for 24 sessions.

Results: The overall response rate for this regimen was $90 \%$, with four patients $40 \%$ achieving complete regrowth of hair (100\%). Three patients have exhibited a satisfactory response (>70\% regrowth). Unsatisfactory response ( $>10-<70 \%$ regrowth) was reported in two patients. Younger patients responded better, as did those with a shorter history of the disease $P<0.05$. At follow-up, which continued for 8-12 months, recurrence was noted in two $(22.2 \%)$ of the nine responders.

Conclusions: Combining excimer phototherapy with triamcinolone acetonide showed a promising effect on resistant AT. This treatment modality was effective and well tolerated particularly in young patients.

Keywords: Alopecia totalis, excimer light, Triamcinolone acetonide, Combined treatment

*Correspondence to Author:

Nabeel K. Alhamzawi

Department of Dermatology, Diwaniyah Teaching Hospital, Diwaniyah, Iraq

How to cite this article:

Nabeel K. Alhamzawi.Combining 308-monochromatic excimer phototherapy with monthly IM triamcin-olone acetonide for the treatment of resistant alopecia totalis. American Journal of Dermatological Research and Reviews, 2021, 4:37.

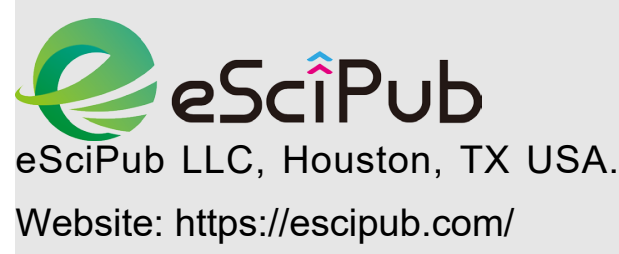




\section{Introduction:}

Approximately $2 \%$ of the world population has alopecia areata $(A A)$, but its prevalence significantly differs by region ${ }^{[1]}$. Alopecia totalis (AT) is a severe variety of alopecia with more dramatic features and a poor prognosis [2]. Refractory alopecia totalis can be defined as alopecia with unsatisfactory response to systemic treatment or topical immunotherapy. Various genetic and environmental factors may influence the pathophysiology of the disease and cause hair loss in alopecia patients. Hair loss in AA is caused by

lymphocytic infiltrations, IFN- $Y$, and IgG antibodies against the hair follicles. The disease may coexist with other autoimmune disorders ${ }^{[3]}$. Treating AT is a major challenge for clinicians in daily practice. Several therapeutic modalities have been explored, but none are currently approved by the Food and Drug Administration. Treatments studied for AA include topical immunotherapy, systemic steroids, and immunosuppressive agents such as cyclosporine and methotrexate, sulfasalazine, PUVA, Janus kinase inhibitors, and TNFa inhibitors. However, no treatment has been found completely effective in significant hair growth. Among these options, topical immunotherapy with diphenylcyclopropenone and squaric acid dibutyl ester have shown encouraging results, but still, need further study ${ }^{[4]}$. This study describes the efficacy and safety of combining monthly pulses of triamcinolone acetonide and 308-excimer phototherapy for treating AT.

\section{Subjects and Methods:}

\section{Subjects}

A prosprctive interventional study evaluated ten patienst with alopecia totalis treated with 308excimer phototherapy and systemic corticosteroids during 2017 and 2018. The patients had not received any treatment in the last six months. Before commencing systemic steroids, a thorough clinical examination was carried out to assess the general health condition and presence of comorbidities such as hypertension, diabetes mellitus, peptic ulcer, or other serious medical illness. Laboratory analysis including $\mathrm{CBC}$, thyroid function test, HbA1c, ANA, LFT, RFT, and VDRL test was performed. The study was conducted according to the World Medical Association's Declaration of Helsinki. Institutional ethical approval was given and consent was obtained from all patients.

\section{Materials}

Irradiation during this study used a 308-nm excimer lamp (Exciplex XeCL; CLARTEIS, France), category $\mathrm{llb}$, spot size $25 \mathrm{~cm}^{2}(5 \times 5)$, with an influence density of $100 \mathrm{~mJ} / \mathrm{cm}^{2}$ and maximum fluence of $3,000 \mathrm{~mJ} / \mathrm{cm}^{2}$. Triamcinolone acetonide $40 \mathrm{mg} / \mathrm{mL}$ (BristolMayers Squibb) was injected intramuscularly every month for each patient.

\section{Treatment protocol}

The affected skin was irradiated by exposure to excimer phototherapy light, starting with 300 $\mathrm{mJ} / \mathrm{cm}^{2}$. The procedure was done twice weekly with an increment dose of $50 \mathrm{~mJ}$ for each session until erythema was exhibited. The maximum dose was $600-900 \mathrm{~mJ} / \mathrm{cm}^{2}$. Intramuscular injection of triamcinolone acetonide (TAC) was added to the treatment at the four-week point for a maximum of six doses.

\section{Evaluation}

The patients were followed up monthly and serial photographs were taken to assess the progress of hair growth. The response to the treatment was assessed based on changes in SALT score which was categorized as follow: SALT of $(100 \%$ regrowth) defined as complete, SALT of $(>70 \%$ regrowth) regarded as satisfactory, SALT of (10$70 \%$ regrowth) labeled as unsatisfactory, and SALT of ( $<10 \%$ regrowth) was considered as no response. Relapse defined as re-fall of the new hair after discontinuation of treatment. Adverse effects were determined throughout and after the end of the study.

If regrowth of hair did not occur within two months, the treatment was stopped. Clinical assessment of the adverse effects of 
corticosteroid was done at every visit. The patients were investigated for suprarenal suppression (measured by cortisol level), mineral bone density (measured by DEXA), and blood sugar level. Follow-up continued for 8 to 12 months after the end of treatment to evaluate relapse.

\section{Data analysis}

Data were analyzed using SPSS software version 26, by IBM, Chicago USA. Data were defined as mean, standard deviation, number, and percentage. A paired $t$-test was used to study the rate of changes in the SALT score before and after treatment. Pearson's correlation was used to determine the association between the changes in the SALT score and the following variables(patient age, duration of the disease, and comorbidity), $P<0.05$ was considered significant.

Table 1. Demographic of patients

\begin{tabular}{|l|l|}
\hline$\cdot$ & Data \\
\hline Patient no. & 10 \\
\hline Gender & \\
\hline M/F & $4 / 6$ \\
\hline Age(year) & $19-45$ \\
\hline Mean & 28.2 \\
\hline Age of disease & $16-42$ \\
\hline Onset & \\
\hline Disease duration (year) & $2.2-5.5$ \\
\hline Mean & 3.19 \\
\hline Previous treatment & \\
\hline S. steroid & 6 \\
\hline T. immunotherapy & 1 \\
\hline PUVA & 1 \\
\hline Cyclosporine & 1 \\
\hline T.treatment & 1 \\
\hline Comorbidities & \\
\hline Atopic dermatitis & $1(10 \%)$ \\
\hline Autoimmune & $1(10 \%)$ \\
\hline Family history & $1(10 \%)$ \\
\hline
\end{tabular}

Table 2. Frequency of excimer sessions, maximum dose, cumulative dose, and TAC pulses in this study

\begin{tabular}{|l|l|l|l|l|}
\hline Variance & N & Range & Mean & SD \\
\hline Excimer sessions & 10 & $14-24$ & 19 & 4 \\
\hline Maximum dose/mJ & 10 & $600-900$ & 750 & 120 \\
\hline Cumulative dose/mJ & 10 & $8750-13300$ & 11130 & 1624 \\
\hline TAC & 10 & $4-6$ & 4.9 & 0.87 \\
\hline
\end{tabular}


Table 3. The changes in the SALT score concerning the age, gender, duration of AA, and comorbidities of the patients.

\begin{tabular}{|l|l|l|l|l|l|}
\hline Patient no. & Age/Gender & disease duration & comorbidity & changes in SALTs & category of response \\
\hline 1 & $19 / \mathrm{F}$ & 2.2 & - & $100 \%$ & complete \\
\hline 2 & $22 / \mathrm{F}$ & 2.4 & - & $100 \%$ & complete \\
\hline 3 & $28 / \mathrm{M}$ & 2.5 & - & $100 \%$ & complete \\
\hline 4 & $25 / \mathrm{F}$ & 2.4 & - & $100 \%$ & complete \\
\hline 5 & $26 / \mathrm{M}$ & 2.8 & - & $90 \%$ & satisfactory \\
\hline 6 & $24 / \mathrm{M}$ & 3.2 & - & $82 \%$ & satisfactory \\
\hline 7 & $34 / \mathrm{F}$ & 3.4 & - & $80 \%$ & satisfactory \\
\hline 8 & $45 / \mathrm{F}$ & 3.5 & + & $42 \%$ & unsatisfactory \\
\hline 9 & $24 / \mathrm{M}$ & 4 & + & $50 \%$ & unsatisfactory \\
\hline 10 & $35 / \mathrm{F}$ & 5.5 & + & $5 \%$ & poor \\
\hline
\end{tabular}

Table 4. The rate of hair regrowth according to the number of excimer and TAC sessions.

\begin{tabular}{|l|l|l|l|}
\hline & M/F & No. of excimer sessions & no. of TAC pulse \\
\hline Complete regrowth & $1 / 3$ & $16 / 16,14,20$ & $4 / 4,6,4$ \\
\hline Satisfactory R. & $2 / 1$ & $18,24 / 20$ & $5,5 / 6$ \\
\hline Unsatisfactory R. & $1 / 1$ & $24 / 24$ & $5 / 6$ \\
\hline Poor response & $0 / 1$ & 14 & 4 \\
\hline
\end{tabular}

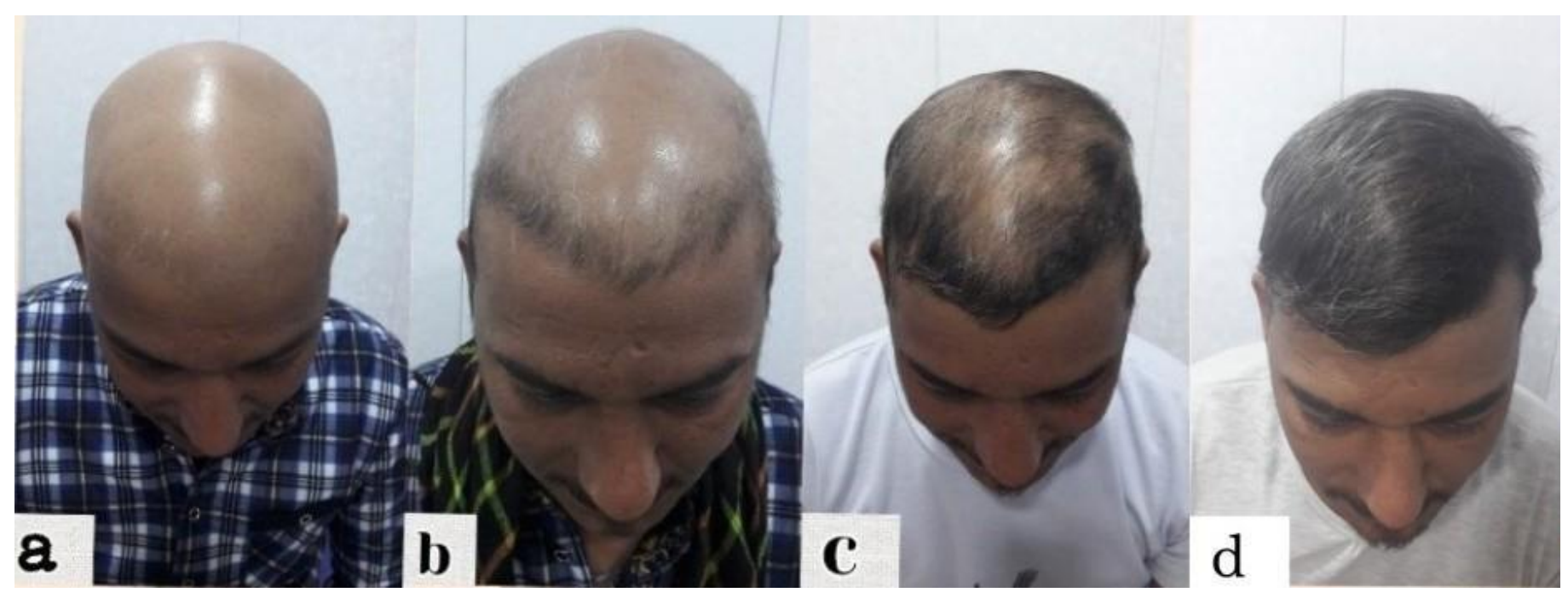

Fig 1. [a] A 28-year old male with AT, [b] 4 weeks after one injection of TAC and 8 excimer sessions, [c] 8 weeks after two injections and 16 excimer sessions, [d] complete regrowth of hair 16 weeks after he received 4 TAC injections and 16 excimer sessions. 


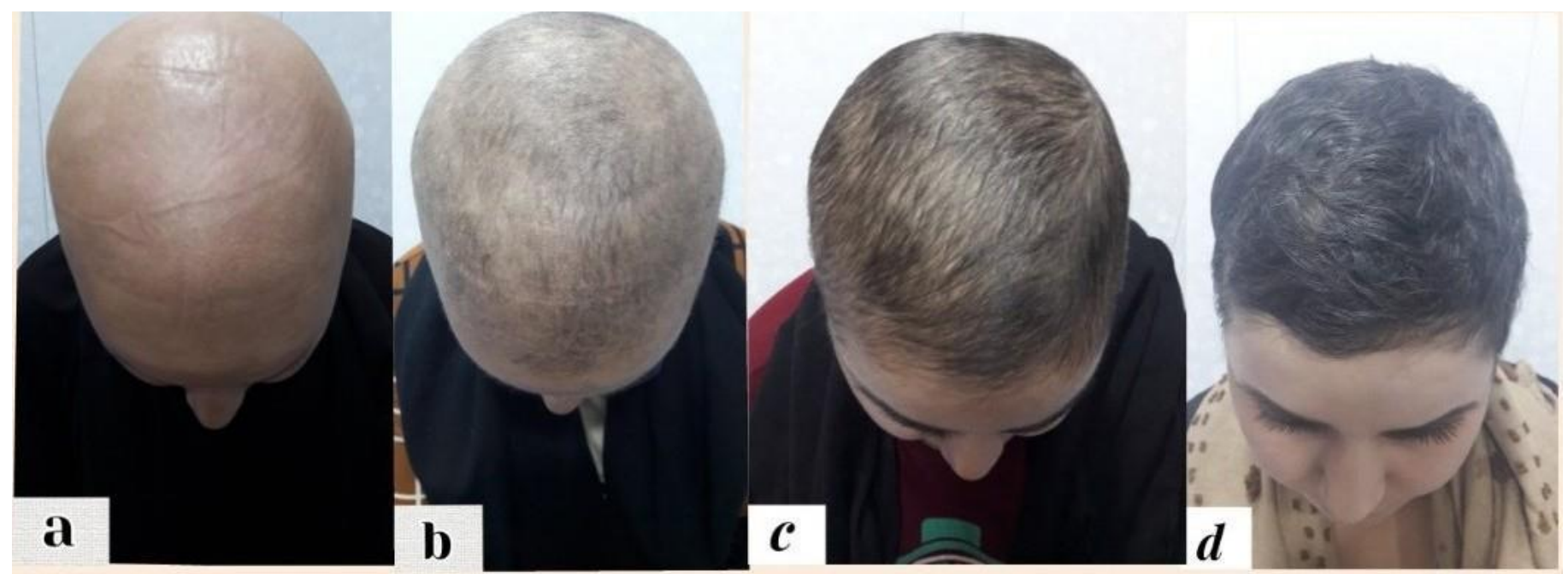

Fig. 2 a. A 22-year old female with alopecia totalis, b. 4 weeks after she received one injection of TAC and 8 excimer sessions, $c 8$ weeks after two injections and 16 excimer sessions, $d$ complete regrowth of hair after 16 weeks of 4 injections and 16 excimer sessions.

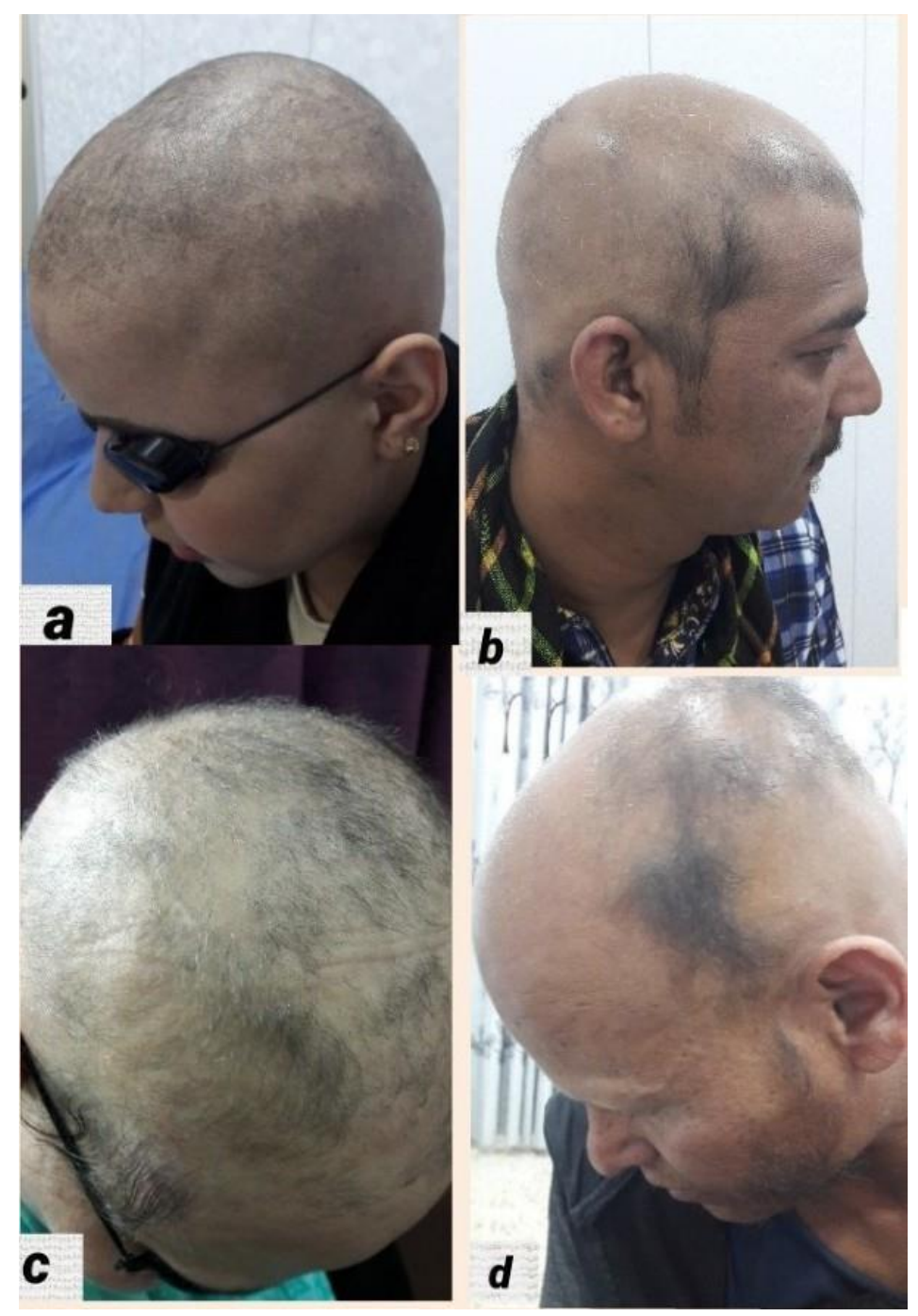

Fig 3. Distribution of new hair in different manners [a] uniform, [b] peripheral [c] scattered, and [d] central. 



Fig. 4 The significant changes in SALT score in relation to the ages of the patients, $P<0.05$. Disease duration has been significantly correlated with the comorbidity $p<0.007$.

\section{Results:}

Ten patients with AT were recruited in this study: four males and six females aged (range 19-45 years; mean $28.2 \pm$ standard deviations [SD] 7.71). The duration of the disease ranged from 2.2- 5.5 years; (mean $3.19 \pm$ [SD] $0.9)$, and the age of disease onset from 16-42 years. Eight patients had type 4 Fitzpatrick skin, and two patients had type 3 skin. Family history was positive in one female patient, and comorbidities were reported in two patients (Table 1). The total number of excimer sessions required to achieve complete regrowth ranged from 14 to 24 , with a mean of $19 \pm$ [SD] 4. The maximum irradiation dose ranged between 600 to $900 \mathrm{~mJ} / \mathrm{cm}^{2}$ with a mean of $750 \mathrm{~mJ} / \mathrm{cm}^{2}$. Regarding triamcinolone acetonide, the number of pulses that required to achieve regrowth of hair without significant adverse reactions ranged from 4 to 6 , mean $4.9 \pm$ [SD] 0.87 (Table 2).

The overall response rate for this regimen in treating AT was $90 \%$, with four patients $40 \%$ (three females and one male) achieving complete regrowth of hair (100\%). Three patients (two male and one female) were achieved satisfactory response ( $>70 \%$ regrowth). Unsatisfactory response ( $>10-<70 \%$ regrowth) was reported in two patients. One female patient was failed to respond to treatment in two months, so the treatment was stopped (Table 3). The last resistant case received 14 excimers sessions and four TAC injections (Table 4).

In two of the patients with complete regrowth, the improvement was noted after four weeks of treatment [ Fig. 1 and 2]. In patients who showed $70-100 \%$ regrowth, the new hair began to appear in the center of the scalp in five patients, and peripherally in one. The new hair initially distributed in several manners: uniform (two patients), central (two patients), scattered (one patient), and peripheral (one patient) [Figure 3]. There was a significant correlation between the age of the patients and the changes in the SALT score, young patients responded better than older group $P$ $<0.05$. Furthermore, there was a strong 
correlation between comorbidity and duration of the disease, $P<.007$ [Fig. 4].

Adverse effects were recorded in two patients $(20 \%)$, including tender erythema and desquamation of the skin. These effects were temporary and resolved after the cancellation of the subsequent session. At the follow-up period, recurrence was noted in two (22.2\%) of the nine responders.

\section{Discussion:}

Alopecia totalis is an advanced form of hair loss with an unpredictable response to treatment. Current evidence for any therapeutic regimen against it is limited and based on small, nonrandomized controlled trials. Therefore, more effort is required toward finding a new treatment regimen that achieves better outcomes with minimum adverse reactions. The use of systemic pulse corticosteroid therapy for AA was first introduced in 1974, to minimize the side effects associated with prolonged systemic corticosteroids ${ }^{[5]}$. Since then, many studies have tried different regimens including pulse intravenous methylprednisolone and pulse intravenous dexamethasone for treating AA, with a variable response and relapse rates ${ }^{[6,7,8]}$. Some studies have described the use of pulse intramuscular triamcinolone acetonide to manage extensive AA and AT, with a moderate response and acceptable level of side effects ${ }^{[9,10]}$. Moreover, relapse has been noted in $46.5 \%$ of responders ${ }^{[10]}$.

\section{Excimer phototherapy has been used} successfully for treating multiple $A A$ and a few cases of AT, for its high-dose monochromatic UV radiation that can trigger apoptosis and induce immunological suppression ${ }^{[11,12,13]}$. The present study relied on combining intramuscular triamcinolone acetonide with excimer phototherapy to obtain a greater synergistic effect. Additionally, this combination aims to reduce the side effects of prolonged use of systemic steroids and to reduce the number of excimer sessions, which become boring once prolonged.
When comparing the results of this study with other studies that used either triamcinolone acetonide or excimer light separately. It was found that the satisfactory response was considered high $(70 \%)$ in this study, compared to $(63 \%)$ in the study used pulse IM (TAC) alone reported by Seo $\mathrm{J}$ et al ${ }^{[9]}$, and (36.4\%) in the study used excimer light alone reported by Arakawa et al ${ }^{[13]}$. Also, the relapse rate in this study (22.2\%) was considered much lower than what was found in the previous study used IM triamcinolone acetonide alone. Furthermore, complete regrowth of hair was achieved in $40 \%$ of patients, suggesting that this modality could become a promising therapy for AT. To conduct this trial, the Exciplex excimer lamp device was chosen, due to its large spot size $(5 \times 5 \mathrm{~cm})$ that helps to speed up the treatment process. Some patients responded to the treatment after a few sessions, which means that the response rate was not related to the number of sessions or the irradiated dose. The new hair appeared in several manners (uniform, central, scattered, and peripheral). These variations between patients in the geographical distribution of new hair might relate to the history of the disease and the immune status of each person. Younger patients responded better, as did those with a shorter history of the disease. This may be what was seen in the two females who achieved complete regrowth (100\%), in whom the history of the disease was 2.2 years and 2.5 years, respectively. Advanced investigations of the patient who did not respond to treatment found that she had associated atopy. This leads to the conclusion that associated diseases, particularly immunological, may adversely affect the response to the treatment. Regarding the side effects of systemic steroids, only one female reported dysmenorrhea, and no significant suprarenal suppression, bone loss, or altered blood sugar level was detected.

To our knowledge, this is the first study to use excimer phototherapy with systemic corticosteroids for treating AT. Its limitation is that the sample size was too small to generalize the 
results. Randomized controlled trials with large sample sizes are needed.

\section{Conclusion:}

Combining excimer phototherapy with triamcinolone acetonide showed a promising effect on refractory AT. This regimen may be successfully used in patients where potential benefits outweigh the risks.

\section{References:}

1. Lee $H H$, Gwillim E, Patel KR, Hua $T$, et al. Epidemiology of Alopecia Areata, Ophiasis, Totalis, and Universalis: A systematic review and meta-analysis. Journal of the American Academy of Dermatology. 2020 Volume 82, Issue 3, 675 682

2. Burroway B, Griggs J, Tosti A. Alopecia Totalis and Universalis long-term outcomes: a review. J Eur Acad Dermatol Venereol. 2019 Oct 8. doi: $10.1111 / \mathrm{jdv}$.

3. Simakou T, Butcher JP, Reid S, Henriquez FL. Alopecia areata: A multifactorial autoimmune condition. J Autoimmun. 2019 Mar 98:74-85.

4. Kassira S, Korta DZ, Chapman LW, Dann F. Review of treatment for alopecia totalis and alopecia universalis. Int J Dermatol 2017 Aug; 56(8): 801-810.

5. Burton, J. and Shuster, S. Large doses of glucocorticoid in the treatment of alopecia areata. Acta Derm Venereol. 1974; 55: 493-496.

6. Seiter S, Ugurel S, Tilgen W, Reinhold U. Highdose pulse corticosteroid therapy in the treatment of severe alopecia areata. Dermatology. 2001; 202(3):230-4.

7. Tsai YM, Chen W, Hsu ML, Lin TK. High-dose steroid pulse therapy for the treatment of severe alopecia areata. J Formos Med Assos. 2002; 101(3):223-6.

8. Vijayakumar M, Thappa DM. Dexamethasone pulse therapy for extensive alopecia areata. To use or not to use. Indian J Dermatol Venereol Leprol 2002; 68: 52-3.

9. Seo J, Lee YI, Hwang S, Zheng Z, Kim DY. Intramuscular triamcinolone acetonide: An undervalued option for refractory alopecia areata. J Dermatol. 2017; 44(2): 173-179.

10. Kurosawa M, Nakagawa S, Mizuashi M, Sasaki $Y$, Kawamura M, Saito M, Aiba S. A comparison of the efficacy, relapse rate and side effects among three modalities of systemic corticosteroids therapy for alopecia areata. Dermatology 2006; 212(4): 361-5.

11. Ohtsuki A, Hasegawa T, Komiyama E, Takagi A, Kawasaki J, Ikeda S. 308-nm Excimer Lamp for the Treatment of Alopecia Areata: Clinical Trial on 16 Cases. Indian J Dermatol. 2013 Jul;58(4):326.

12. Al Hamzawi NK. Evaluation of the Efficacy and Safety of 308-nm Monochromatic Excimer Lamp in the Treatment of Resistant Alopecia Areata. Int J Trichology. 2019 Sep-Oct;11(5):199-206.

13. Arakawa $\mathrm{Y}$, Nomiyama $\mathrm{T}$, Katoh $\mathrm{N}$. Three hundred and eight-nanometer excimer light therapy for alopecia universalis that is resistant to other treatment: A clinical study of 11 patients. J Dermatol 2016; 43(12): 1412-1416. 